\title{
Magnetically Coated Bioabsorbable Stents for Renormalizaton of Arterial Vessel Walls after Stent Implantation
}

\author{
J.S. Lee ${ }^{1}$, P, Han $^{2}$, H. Song ${ }^{1}$, D. Kim ${ }^{1,6}$, H. Lee ${ }^{2}$, M. Labowsky ${ }^{7}$, S. Ylä-Herttuala ${ }^{8}$, J. Hytönen ${ }^{8}$, \\ M. Gülcher ${ }^{9}$, A.J. Sinusas ${ }^{4,5}$, J. Martin $^{5}$, A. Mathur ${ }^{10}$, T.M. Fahmy ${ }^{1,2,3,{ }^{*}}$. \\ Departments of ${ }^{1}$ Biomedical Engineering, ${ }^{2}$ Chemical and Environmental Engineering, \\ ${ }^{3}$ Immunobiology, ${ }^{4}$ Internal Medicine, and ${ }^{5}$ Cardiovascular Medicine, Yale University, New \\ Haven, CT 06511, USA. \\ ${ }^{6}$ Department of Pharmaceutical Sciences, Irma Lerma Rangel College of Pharmacy, Texas A\&M \\ Health Science Center, College Station, TX 77843, USA. \\ ${ }^{7}$ Ansama Research LLC, Wayne, NJ 07470, USA. \\ ${ }^{8}$ A.I. Virtanen Institute for Molecular Sciences, University of Eastern Finland, Kuopio, 70210, \\ Finland. \\ ${ }^{9}$ QualiMed Innovative Medizinprodukte GmbH, Winsen, 21423, Germany. \\ ${ }^{10}$ Department of Clinical Pharmacology, William Harvey Research Institute, Queen Mary \\ University of London, London E1 4NS, UK.
}




\section{Abstract}

The insertion of a stent in diseased arteries is a common endovascular procedure that can be compromised by the development of short and long-term inflammatory responses leading to restenosis and thrombosis respectively. While treatment with drugs, either systemic or localized, have decreased the incidence of restenosis and thrombosis these complications persist and are associated with a high mortality in those that present with stent thrombosis. We reasoned that if stents could be made to undergo accelerated endothelialization in the deployed region, then such an approach would further decrease the occurrence of stent thrombosis and restenosis thereby improving clinical outcomes. Towards that objective, the first step necessitated efficient capture of progenitor stem cells, which eventually would become the new endothelium. To achieve this objective, we engineered intrinsic ferromagnetism within non-magnetizable, biodegradable Magnesium (Mg) bare metal stents. Mg stents were coated with biodegradable polylactide (PLA) polymer embedding magnetizable iron-Platinum (FePt) alloy nanoparticles, Nano-Magnetic" particles- ${ }^{\text {("n }}$ Mags, which increased the surface area and hence magnetization of the stent. ${ }^{\mathrm{n}}$ Mags uniformly distributed on stents enabled capture, under flow, up to $50 \mathrm{ml} / \mathrm{min}$, of systemically injected iron oxide (IO) labeled progenitor stem cells. Critical parameters enhancing capture efficiency were optimized and we demonstrated the generality of the approach by showing that ${ }^{\mathrm{n}}$ Mag-coated stents can capture different cell types. Our work is a potential paradigm shift in engineering stents because implants are rendered as tissue in the body and this, "natural stealthiness" reduce or eliminate issues associated with pro-inflammatory immune responses post-implantation.

Keywords: ferromagnetic, paramagnetic, drug-eluting stent, nanoparticles, cell therapy, cardiovascular disease, Iron-Platinum, magnetization. 


\section{Introduction}

In 2016, more than 0.75 million stents were implanted worldwide to remedy atherosclerotic cardiovascular diseases and over 0.33 million in the United States alone ${ }^{1}$. In-stent restenosis, however, is a frequent unwanted side effect originating with an inflammatory immune response that attempts to restore immune competence and limit damage in the affected area post foreign material deployment ${ }^{2}$. In addition, because of "tissue scarring", smooth muscle cells proliferate abnormally resulting in narrowing of the vascular lumen around the inserted stent. Conventional treatment for these complications entail the use of anti-mitotic agents coating the stent to prevent restenosis and anti-platelet medications such as Clopidogrel (Plavix), Prasugrel (Effient), Ticagrelor (Brilinta), Ticlopidine (Ticlid) to prevent thrombosis. Use of these medications can result in unwanted side effects, and are rendered less than optimally effective due to patient non-compliance to the drug regimen., ${ }^{3}$ For those reasons, drug eluting stents have raised hopes since they provision a drug locally to the affected region, minimizing side effects, and in a sustained manner, facilitating automatic patient compliance. ${ }^{5}$ However, the main complication have been the long-term effects such as late thrombosis and delayed endothelialization which, collectively, may result in myocardial infarctions. ${ }^{6}$ For example, it has been reported that stent thrombosis develops in a significant fraction of patients implanted with Drug Eluting Stents ${ }^{7}$ leading to delayed arterial healing. ${ }^{8,9}$ Thus, to achieve long-term protection, a new methodology is needed that inhibits restenosis in the short-term and abrogates long term complications like thrombosis. A methodology that renormalizes the vasculature after stent implantation would serve this objective and such an approach would need to begin with a means to achieve rapid and efficient seeding of the affected area with endothelial progenitor stem cells.

Stent endothelialization here refers to capture, growth or proliferation of a fresh layer of endothelial cells on the stent without abrogating its mechanical function. ${ }^{10}$ This can be achieved using administered endothelial stem cell progenitors. ${ }^{11}$ This hypothesis is supported by prevailing thoughts that delivery and localization of progenitor stem cells to the stent implant site may quickly regenerate the endothelium and prevent narrowing of the vascular lumen. ${ }^{12}$ Early efforts aimed at rapid endothelialization involved precoating stents with cultured cells or capturing with ligands or antibodies matured endothelial cells intravenously inoculated allowing for the attachment of those cells and their subsequent differentiation and proliferation. ${ }^{13,14}$ 
Pioneering work on endothelialization was performed as early as the late 1970s, when Herring et al. first reported on autologous endothelial cell seeding. ${ }^{15}$ Endothelial cell seeding was shown to be effective in reducing graft thrombogenicity and intimal hyperplasia in large animal models ${ }^{16}$ and promising results were reported by Ortenwall in human trials. ${ }^{17}$ These techniques, however, are still facing inefficiency in cell retention and non-specific capture of inflammatory cells within the target site. ${ }^{18}$

A potential strategy for achieving complete coverage of the implanted stent with a functional endothelial layer is to provide rapid, uniform, and specific cell recruitment using external forces guided, for example, by force fields such as magnetic fields. This technology relies on rendering cells magnetically responsive, with methods such as labeling with Iron Oxide nano or microparticles. The feasibility of this approach has been demonstrated by several groups showing IO labeled cells can be targeted to paramagnetic medical devices using locally/remotely applied magnetic fields. Paramagnetic stents (i.e; instantly magnetized in the presence of a strong magnetic flux, and demagnetized instantly in its absence), are limited in their application by the insufficient magnetic force gradients experienced by deeper areas of the body, such as coronary vessels where stenting may take place. Furthermore, the inconvenient aspect (a large magnet needs to be available for the procedure) makes this a less attractive option for outpatient or point-of-care clinical settings, which are often not equipped with sophisticated MRI or large magnetic flux equipment.

An optional methodology is to introduce an intrinsic magnetic moment within the stent, i.e., ferromagnetic. Pislaru et al. tried this approach using stainless steel interspaced with for synthetic vascular grafts Others, such as Uthamaraj et al, used stainless steel stents rendered ferromagnetic to capture IO-labeled endothelial cells. ${ }^{19}$ Stainless steel, however, is not biodegradable and thus require long-term drug therapy with antibiotics or antiplatelet therapy. to minimize complications. Further, non-bioabsorable stents prevent lumen expansion, impair vessel geometry and often lead to obstruction of blood vessel side branches. ${ }^{20}$

Magnesium bare metal stents, on the other hand, are bioabsorbable. In addition, $\mathrm{Mg}$ introduces additional features which make it a very attractive choice for stent manufacture. $(\text { Table } 1)^{21}$ First, the use of polymeric coatings such as PolyLactide acid (PLA), or Polylacticco-glycolic (PLGA) results in release of acidic monomers during the degradation process 
lowering the $\mathrm{pH}$ in and around the sten. An acidic microenvironment will impede cell survival and thus the development of a functional endothelial cell layer. $\mathrm{Mg}$ is known to impart an acid neutralizing effect, which is why several antiacid and gastric reflux medications such as Mylanta and Milk of Magnesia have $\mathrm{Mg}$ as the active ingredient. ${ }^{22,23} \mathrm{Mg}$ will therefore improve cell viability in the presence of degradable polymer coatings. ${ }^{24}$ Second, $\mathrm{Mg}$ has anti-inflammatory immune properties, leading to, for example reduction in IL-6 and IL-13. ${ }^{25,}{ }^{26}$ Third, Mg alloys show excellent anti-platelet deposition and low thrombogenicity. ${ }^{27,28}$ Applications in the field of bone regenerative medicine, for example, show that $\mathrm{Mg}$ screws can promote human bone marrow stem cell (hBMSC) differentiation and proliferation ${ }^{29}$ and the same for Mg scaffolds in bone regeneration. Finally, there is evidence of enhanced antioxidant enzyme activity following $\mathrm{Mg}$ supplementation. ${ }^{30}$ Table 1 below, summarizes these features and properties associated with the use of Mg. These properties together with the biodegradability aspect of the material and the engineering of an intrinsically magnetic moment thus offer an unprecedented stent technology that facilitates renormalization of vascularity after stent implantation.

Table 1. Summary of Features Associated with use of $\mathrm{Mg}$ as the stent base material

\begin{tabular}{l|l|c}
\hline Property & Beneficial Impact & Ref. \\
\hline Bioabsorbability & $\begin{array}{l}\text { Minimizes host foreign body response, endothelial cell } \\
\text { dysfunction and thus reduces or abrogates the need for long- } \\
\text { term drug regimens post stent deployment. }\end{array}$ & 7 \\
\hline $\begin{array}{l}\text { Acid- } \\
\text { neutralization }\end{array}$ & $\begin{array}{l}\text { Improves cell viability in the presence of degrading polymeric } \\
\text { (PLA or PLGA) coatings. }\end{array}$ & $22-24$ \\
\hline Anti-inflammation & $\begin{array}{l}\text { Reduces release of pro-inflammatory cytokines such as IL-6 } \\
\text { and IL-13. Reduced fibrosis and platelet deposition and thus } \\
\text { lowering potential thrombogenicity }\end{array}$ & $25-28$ \\
\hline Anti-Oxidant & Reduced oxidative stress and lowering of free radicals & 30 \\
\hline
\end{tabular}

We developed a novel bioabsorbable ferromagnetic stent as a platform to recruit cells tagged with IOs. We note that IO labelling of cells is an already FDA approved, used for tracking cells by MRI. ${ }^{31}$ and commercial kits are available that tag human cells with IO for separation and concentration. Our technology combines the use of conventional bioabsorbable Mg metal stents with an ${ }^{\mathrm{n}}$ Mag-embedded PLA polymer coating ( ${ }^{\mathrm{n}}$ Mag stent) yielding a uniform 5 
stent magnetic moment that was capable of attracting cells under flow. Because, $\mathrm{Mg}$ has been used to fabricate stents with minimal degree of thromboembolic events and inflammation, ${ }^{28}$ we hypothesized that ${ }^{\mathrm{n}} \mathrm{Mag}-\mathrm{Mg}$ stents will capture cells and cover the stent with progenitor stem cells and that effect will enhance stent patency and reduce or eliminate complications over the long-term.

\section{Results and Discussion}

The magnetic force due to surface incorporated ${ }^{n}$ Mag: Rationale for use of nanoparticles and choice of FePt as the magnetizable alloy ("Mags). The intrinsic magnetic moment of a stent would need to be sufficiently strong to capture cells flowing within distances that may exceed several diameters of the stent itself. We reasoned that a strong magnetic field can be created by coupling magnetic nanoparticles to the $\mathrm{Mg}$ metallic coronary stents (3 $\mathrm{mm}$ diameter, D and $18 \mathrm{~mm}$ length, L) because the magnetic flux density is directly proportional to the surface area of the magnet according to eq. 1, a higher magnetic force can be introduced by coating with nanoparticles.

$$
F_{\text {coated stent }}=N_{n_{\text {Mags }}} \frac{B^{2} A}{2 \mu_{0}}
$$

$\mathrm{F}=$ magnetic force imparted by stent, $\mathrm{B}=$ flux density, $\mathrm{A}=$ surface area, $\mu_{0}=$ permeability of the medium, $N_{n_{\text {Mags }}}=$ number of beads coating the stent, typically several thousand per stent.

For 7000 particles, for example, and assuming a flux density that is unchanged after coating the stent, the ratio of magnetic force of an uncoated to coated would be:

$$
\frac{F_{\text {coated }}}{F_{\text {uncoated }}}=7000\left(4 \pi r^{2}\right)
$$

which is a significant increase in the magnetic field strength solely based on the incorporation of magnetic nanoparticles on the surface.

Diameter or radius of the particles is thus critical for magnetization force. On the nanoscale, Fe alone does not form ordered magnetizable crystals. Hence doping with Pt gives rise to a stable face centered tetragonal (fct) crystal with high magnetic anisotropy. ${ }^{32-34}$ The choice of FePt alloy was thus based on achieving a stable crystal structure alignment of the magnetic dipoles. ${ }^{32} 35$ 
Synthesis and characterization of ${ }^{\mathbf{n}}$ Mags. Monodisperse FePt nanoparticles (NPs) were prepared by chemical reduction of platinum(II) acetylacetonate (Pt(acac) $)_{2}$ ) and iron(III) acetylacetonate $\left(\mathrm{Fe}(\mathrm{acac})_{3}\right)$ (Figure 1a). ${ }^{36}$ We utilized transmission electron microscopy (TEM) and high-angle annular dark-field scanning transmission electron microscopy (HAADF-STEM) to show FePt particles of approximately 1-3 nm diameter (Figure 1d) and co-localization of the $\mathrm{Fe}$ and $\mathrm{Pt}$ elements within individual nanoparticles (Figure 1b). The FePt particles have a chemically disordered face-centered cubic (fcc) structure and can be transformed into an ordered fct structure after proper thermal treatment (annealing) to yield stable ferromagnetic properties. Heat treatment is known to lead to sintering and agglomeration of NPs thus broadening the size distribution and increasing particle size (micrometers) (Figure 1d). To reduce thermally-induced coalescence of FePt NPs, a silicon dioxide coating $\left(\mathrm{SiO}_{2}\right.$ has a high-melting-point, $\left.\mathrm{T}_{\mathrm{m}}: 1713{ }^{\circ} \mathrm{C}\right)$ was used to protect FePt NPs prior to heat treatment. ${ }^{37}$

The compositionally ordered L10 phase of FePt $\mathrm{Np}$ was formed when $\mathrm{SiO}_{2}$ coated FePt NPs were annealed under forming gas $\left(93 \% \mathrm{~N}_{2}+7 \% \mathrm{H}_{2}\right)$ at $650{ }^{\circ} \mathrm{C}$. The $\mathrm{SiO}_{2}$ layer reduced thermal sintering of FePt particles, yielding a mean size of $200 \mathrm{~nm}$ for coated FePt NP (Figure $1 \mathrm{~g})$. Particle sizes in this range are amenable to dispersion in organic solvents, which is critically required for uniform coating of stents with the particles. After annealing, the silica layer was completely removed by washing with HF solution (1\%). Within 1 min after start of the annealing process, a phase transformation can be observed using X-ray diffraction. This phase change imparts the ferromagnetic properties of FePt (Figure 1f).

To assess the magnetic character of the particles, a superconducting quantum interference device (SQUID) was utilized to derive in-plane magnetic hysteresis of the particles (Figures 1c and 1e). SQUID testing showed a high uniaxial magnetocrystalline anisotropy which is responsible for the measured coercivity of approximately $1.0 \mathrm{~T}$ post-annealing. Henceforth, the magnetic FePt particles of size $300 \mathrm{~nm}$ and an average coercivity of 1.0 T were termed, "'Mags", to reflect their nanoscale ferromagnetic properties. To induce permanent ferromagnetization, ${ }^{\mathrm{n}}$ Mags were placed in a $1.5 \mathrm{~T}$ magnetic field solenoid overnight. We note, that this solenoid is the same as that typically used for human MRI tests. We also observed that magnetization can be induced in less than an hour in the same magnet (data not shown), after which the characteristic, 
dispersible black particle powder agglomerated into clusters as a result of the magnetization of individual particles which then attracted each other (Figure 1h).

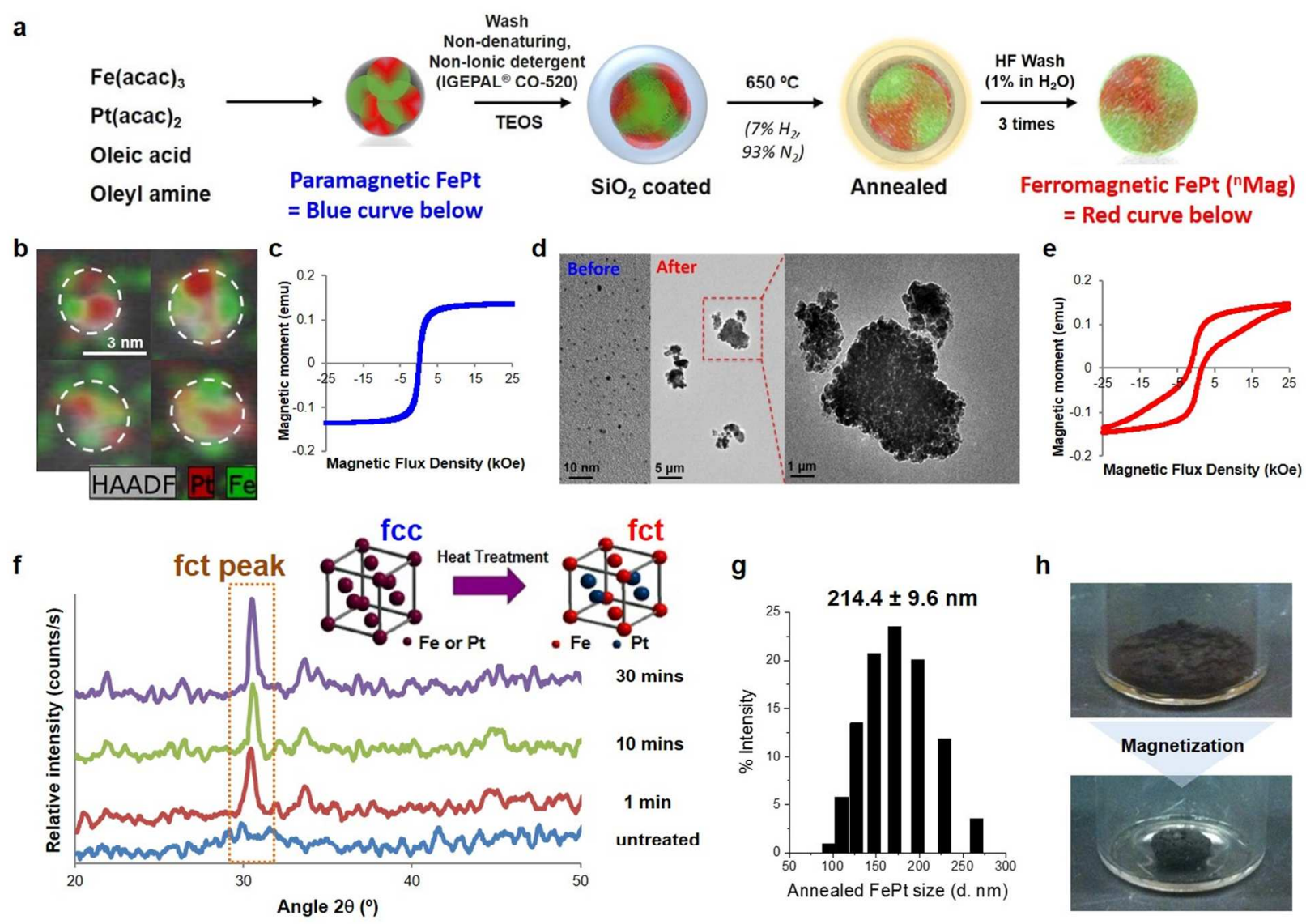

Figure 1. Synthesis and characterization of magnetizable FePt NPs ( ${ }^{\mathrm{n}}$ Mags). (a) Synthetic process involving reaction of the constituent elements to form FePt NPs, followed by chemical reduction, coating with $\mathrm{SiO}_{2}$, annealing at $650^{\circ} \mathrm{C}$ then silica coat removal by an $\mathrm{HF}$ wash. (b) Demonstration of the blending and localization of Fe and Pt in nanoparticles using High-angle annular dark-field scanning transmission electron microscopy (HAADF-STEM). (c) Paramagnetism. A hysteresis in the magnetization loop of non-annealed NPs. (d) Transmission electron microscopy (TEM), showing FePt particles of approximately 1-3 nm diameter. (e) Ferromagnetism. A hysteresis in the magnetization loop demonstrates induction of permanent magnetic dipole moments (magnetic memory) in annealed NPs. The hysteresis is responsible for the high uniaxial magnetocrystalline anisotropy leading to coercivity. (f) Assessment of magnetic anisotropy using X-ray diffraction (XRD). XRD patterns show transition of FePt alloy NPs from 
chemically disordered, low magnetic anisotropy (fcc) to, ordered high magnetic anisotropy (fct) structure (peaks shown enclosed by red dotted square) as a function of the annealing time. (g) Measurement of particle size after annealing using dynamic light scattering (DLS) (mean \pm std.). (h) Simple visual assessment of induction of magnetization. Particles magnetized in a $1.5 \mathrm{~T}$ coil form an aggregate cluster.

Fabrication of the ${ }^{\mathrm{n}} \mathrm{Mag}$ coated Stent. Bare metal Mg stents were made into magnetic stents by spray coating with a polymer-solvent solution composed of the FDA approved poly(lactic acid) (PLA) polymer as the base substrate mixed with ${ }^{\mathrm{n}}$ Mags at a ratio of 30\% by wt. The stent and coating properties were measured using a scanning electron micrographs (SEM): Mg strut thickness: $100 \mu \mathrm{m}$, PLA coating: $30 \mu \mathrm{m}$ (Figure S1). PLA was chosen as the embedding substrate for three reasons: A) controlled hydrolysis-induced biodegradation, B) physio-chemical properties (PLA hydrophobicity closely matched that of the ${ }^{\mathrm{n}}$ Mags, enabling ${ }^{n}$ Mags uniform dispersion and retention within the coat.), C) minimal water absorption, enabling stable coating for at least a couple of weeks after implantation (see Figure S2). The schematic in Figure $2 \mathrm{a}$ depicts the process of electrospraying of the ${ }^{\mathrm{n}}$ Mag-polymer-coated stent. Here ${ }^{\mathrm{n}}$ Mags dispersed in a PLA $\mathrm{CHCl}_{3}$ solution were electrosprayed over the $\mathrm{Mg}$ stent while rotating the stent axially (Details are in the method section.). The PLA coating allowed the addition of ${ }^{\mathrm{n}}$ Mags into the stent coating at a $30 \%$ of total coating mass. The stent coated with ${ }^{\mathrm{n}}$ Mags was magnetized in a $1.5 \mathrm{~T}$ magnetic field overnight, BRUKER MRI Solenoid. While we chose $24 \mathrm{~h}$ to ensure complete dipole alignment, similar results were with magnetization were observed as brief as $1 \mathrm{~h}$ post insertion of the device in the magnetic field (data not shown). Magnetization was evident given the propensity of the device to attract steel objects (Figure 2b).

Figure 2c depicts a rough approximation of the magnetic field lines around a coated cylindrical object, such as a stent. This figure was generated by superimposing the individual fields of some 7000 axially-aligned dipoles symmetrically located inside a cylindrical tube (stent). Strikingly, this rough approximation predicts that the field lines will pull cells to the luminal area of the stent. Note, this field differs from that in the interior of a solenoid in which the field lies are parallel to the cylinder's surface and, thus, would not attract cells to the surface and push the cells towards the ends. A more rigorous future calculation will map the trajectories 
of the magnetized stem cells as they flow through the stent. Therefore, fortuitously, coating with the nanoparticles introduces magnetic forces that "pull" cells toward the luminal side of the stent but also facilitate uniform distribution of those cells across the length of the stent.

a

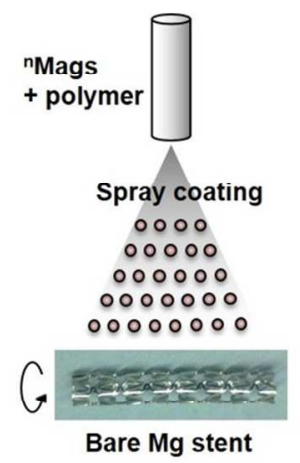

C

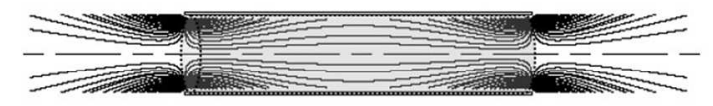

nMag-coated cylindrical device b
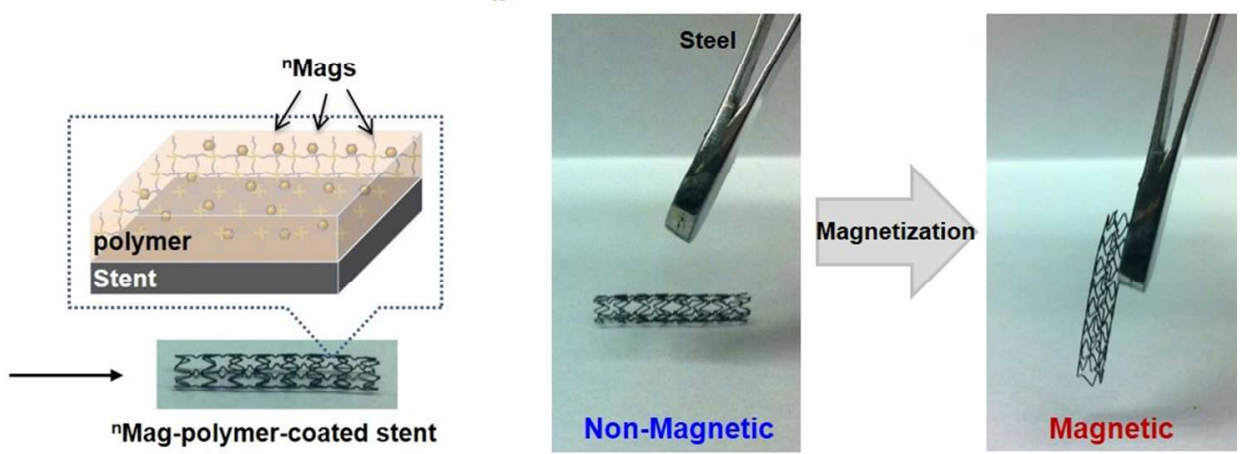

Figure 2. Schematic of the procedure for coating the stent with ${ }^{n}$ Mag. (a) Electrospray deposition of PLA and ${ }^{\mathrm{n}}$ Mags on an axially rotating Mg stent. The process leads to embedding of the ${ }^{\mathrm{n}}$ Mags after coating. (b) Visual assessment of ${ }^{\mathrm{n}}$ Mags stent magnetization. No response was observed before magnetization. (c) Magnetic field lines inside a particle coated cylindrical device.

Cell Capture Efficiency: To quantitate capture efficiency, cells were labeled by either internalizing IO or surface receptor labeled with antibody coated-IO. A schematic of the bioreactor flow set-up is shown in Figure 3a. Under flow of $25 \mathrm{ml} / \mathrm{min}$ we quantitated capture efficiency over time as shown in Figure 3, where cells were injected into $3 \mathrm{~cm}$ away from the ${ }^{n}$ Mag stent. A control stent, polymer-coated stent without ${ }^{n}$ Mags was used to compare. The presence of a magnetic surface generated by ${ }^{n}$ Mags substantially increased cell capture onto the stent (Figure 3b). The percentage of cell capture of ${ }^{\mathrm{n}}$ Mag stent was up to $83 \%$, while it was $12 \%$ for polymer-coated stent (Figure 3c). It was clear that the magnetic field generated by the ${ }^{\mathrm{n}}$ Mags substantially increased cell capture onto the stent even at flows up to $50 \mathrm{~mL} / \mathrm{min}$, corresponding 
to normal physiological blood flow in the proximal coronary artery. This equates to a shear stress of 840 dyne $\cdot \mathrm{s} / \mathrm{cm}^{2}$ (typical shear stress in coronary arteries is $<400 \mathrm{dyne} \cdot \mathrm{s} / \mathrm{cm}^{2}$ ). For these experiments, the fluid shear stress $\left(t, d y n e \cdot s / \mathrm{cm}^{2}\right)$ in the channel was calculated according to the equation:

$$
\mathrm{t}=\frac{6 \mathrm{Q} \mu}{b h^{2}}
$$

where $\mathrm{Q}$ is the flow rate $(50 \mathrm{~mL} / \mathrm{min}), \mu$ is the viscosity of the perfusate $(0.007$ poise $), \mathrm{b}$ is the width of the flow channel $(0.3 \mathrm{~cm})$, and $\mathrm{h}$ is the height of the flow channel $(0.3 \mathrm{~cm})$.

To evaluate degradation, a fluorophore was co-encapsulated in the polymer coating reporting on polymer degradation via fluorophore release from the degraded coating. First, we observed that the presence of ${ }^{n}$ Mags did not impact the integrity of the stent coating. This was demonstrated by comparing the release kinetics of fluorophore from polymer coated stents with and without the ${ }^{\mathrm{n}}$ Mags (Figure S2). Degradation of ${ }^{\mathrm{n}}$ Mag stent was evaluated in a convective flow bioreactor and a slow degradation for up to $35 \mathrm{~d}$ was observed (Figure $3 \mathrm{~d}$ ). No obvious recoiling of stent was found and the original diameter was maintained for up to 3 weeks. 


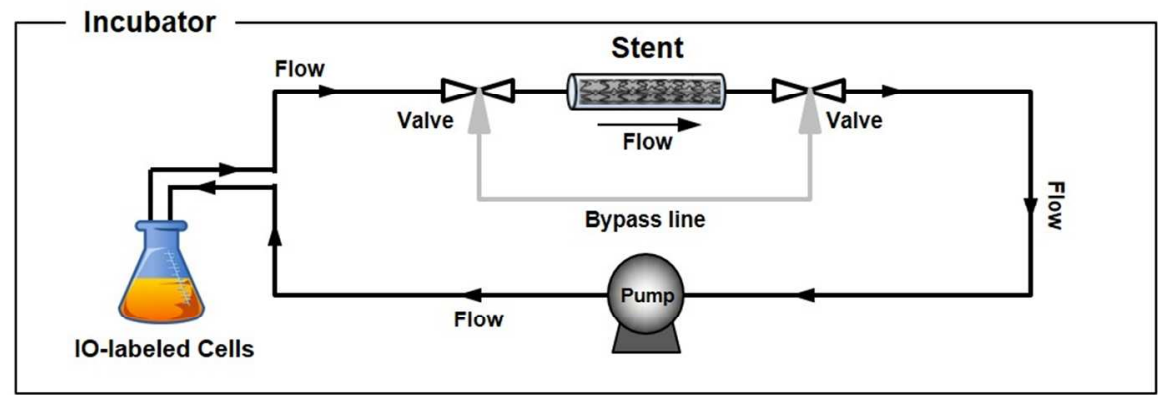

b

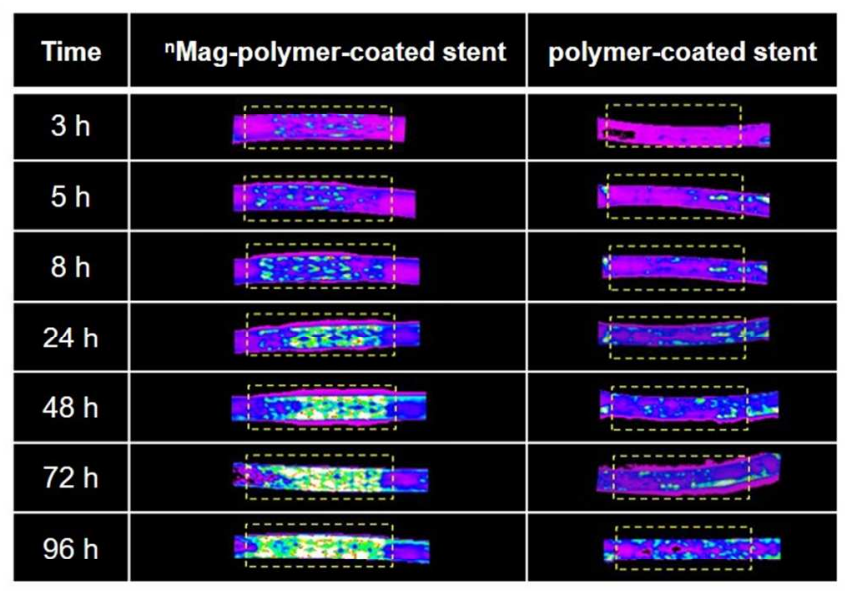

C

d

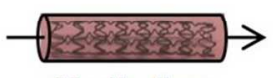

Media flow

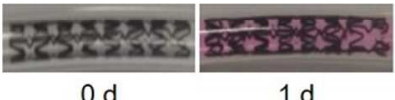

$1 \mathrm{~d}$

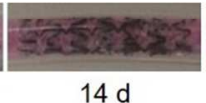

$14 \mathrm{~d}$

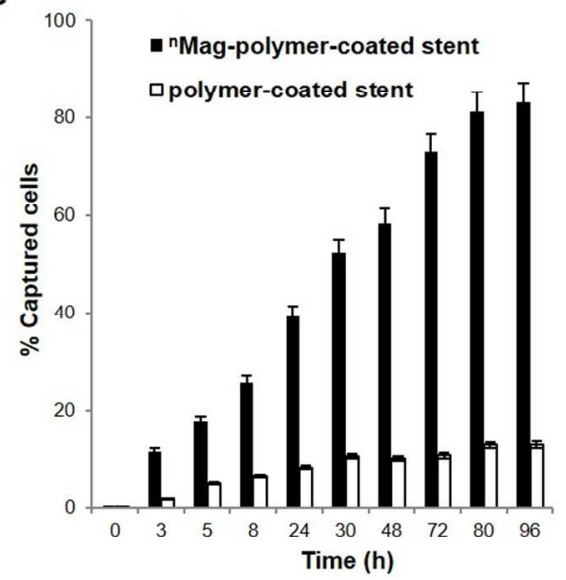

Figure 3. Efficiency of Cell Capture in a circulation flow bioreactor. (a) A flow-loop bioreactor set-up was used to mimic physiological shear flow conditions and efficiency of cell capture under those conditions. Macrophages $\left(1 \times 10^{6}\right)$ that have endocytosed IO particles in the media were used as a first demonstration of general magnetic cell capture. Macrophages were also fluorescently labeled for visualization and quantification of cell capture. (b) Cell capture by the ${ }^{\mathrm{n}}$ Mag stent over time compared to a control stent with polymer but no ${ }^{\mathrm{n}}$ Mags. (c) Quantitative assessment of cell capture over time. (d) The mode of degradation of ${ }^{\mathrm{n}}$ Mag stent was monitored in a flow-loop bioreactor over one month.

Impact of other parameters on cell capture. Critical parameters that impact the capture efficiency include, concentration of ${ }^{n}$ Mags, flow rates (shear stress), and the initial cell number offered for capture. To ascertain the effect of these factors and to establish the generality of the platform in cell capture we repeated capture efficiency studies with Human Umbilical Vein 
Endothlial Cells (HUVECs) which internalized iron-oxide by endocytic uptake (Figure S4). The ${ }^{\mathrm{n}} \mathrm{Mag}$ concentration and shear force were shown to be the most effectual variables on cell capture. At a fixed shear stress of 840 dyne $\cdot \mathrm{s} / \mathrm{cm}^{2}(50 \mathrm{~mL} / \mathrm{min})$, the presence of the ${ }^{\mathrm{n}} \mathrm{Mags}$ on the stent (30 wt\%) yielded a cell capture density of more than $500 \mathrm{cell} / \mathrm{s} / \mathrm{mm}^{2}$, a 37-fold increase in the number of retained cells as compared to a stent with no ${ }^{\mathrm{n}}$ Mags (0 wt\%) (Figure 4a). Cells were also captured more efficiently (Figure $4 b$ ), when the flow rate was reduced by half. In contrast, the number of injected cells did not significantly influence the cell capture efficiency when the initial input number of cells was reduced by a 1/3 (from 1 to 0.3 million) (Figure 4c). Of the captured cells, about 10-20\% were attracted in the first pass through the circulation loop (Figure 4d). Understanding cell capture in the first pass is a critical parameter for translational application since in clinical settings cells are injected proximally to the stent and they then circulate through the body. Thus, first pass cell capture is crucial as it prognosticates the potential success of the device in capturing a sufficient number of cells that will proliferate and ultimately renormalize the impacted region. 
a

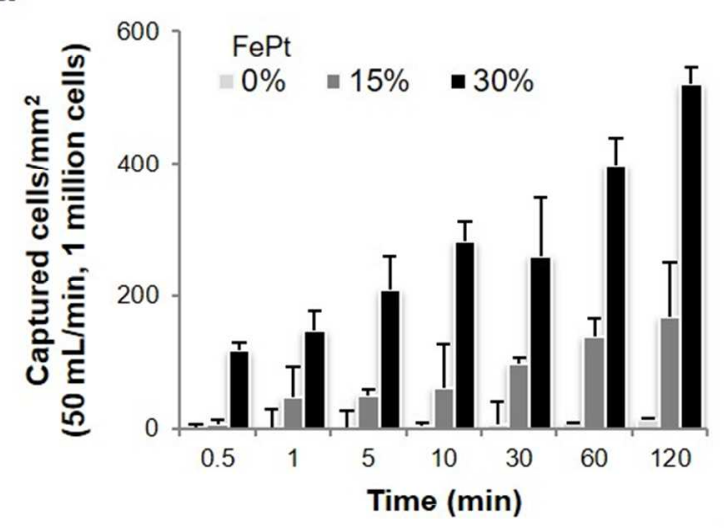

C

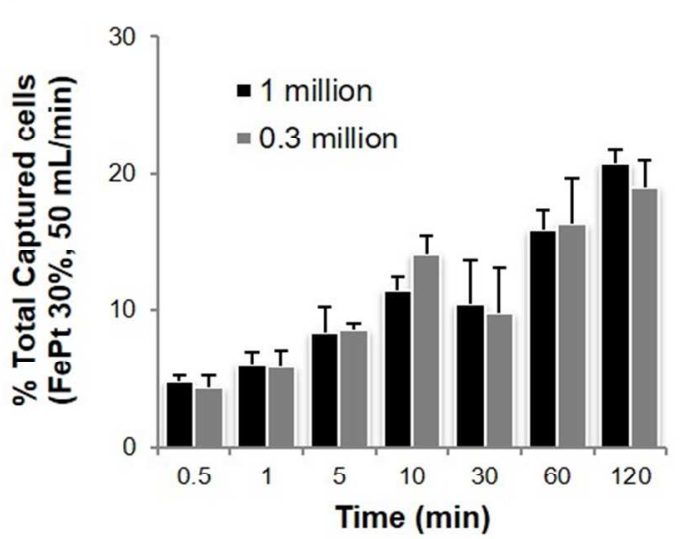

b

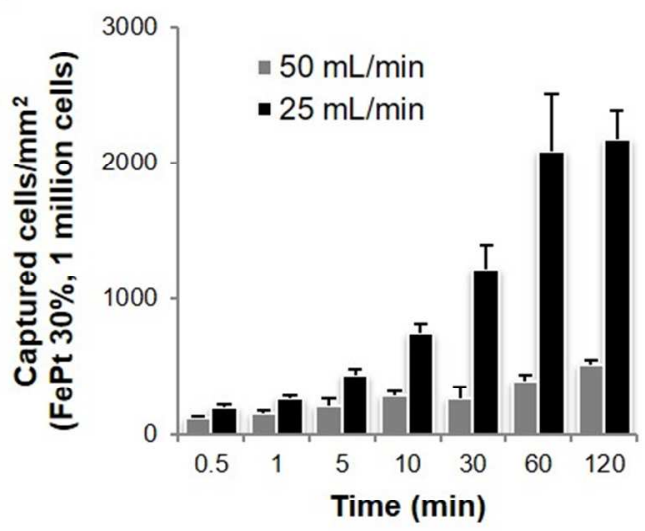

d

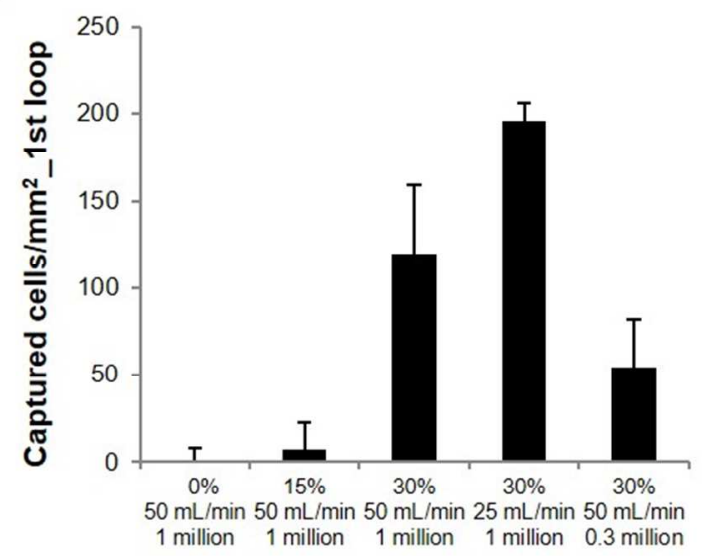

e

\begin{tabular}{|c|c|c|}
\hline Time & nMag-polymer-coated stent & polymer-coated stent \\
\hline $0 \mathrm{~h}$ & & \\
\hline $1 \mathrm{~h}$ & & \\
\hline $3 \mathrm{~h}$ & & \\
\hline $6 \mathrm{~h}$ & & \\
\hline $10 \mathrm{~h}$ & & \\
\hline $17 \mathrm{~h}$ & & \\
\hline $24 \mathrm{~h}$ & & \\
\hline
\end{tabular}

Figure 4. Capture of circulating HUVECs by ${ }^{\mathrm{n}}$ Mag-polymer-coated stent. The effects of (a) ${ }^{\mathrm{n}}$ Mag seeding concentration, (b) flow rate, and (c) the number of cells infused into the stent flow chamber. (d) The number of retained cells after the first pass (the most crucial pass in clinical practice) (e) Visualization of HUVEC retention of HUVECs at $50 \mathrm{~mL} / \mathrm{min}$ and $24 \mathrm{~h}$ using stents loaded with $30 \mathrm{wt} \%{ }^{\mathrm{n}}$ Mags and $0 \%{ }^{\mathrm{n}}$ Mags. 


\section{Clinically relevant flow conditions and their impact on capture of progenitor stem}

cells. To demonstrate the utility of the magnetic stent in stem cell capture for reendothelialization, we next labeled CD34+ human hematopoietic progenitor stem cells (hHSCs) with the clinically approved CliniMACS (a dextran-coated superparamagnetic iron oxide agent) immobilized with anti-CD34 antibodies using manufacturer protocol (supplementary information).

A clinically relevant protocol for HSC cell therapy utilizes what is termed as a "stasis protocol" (Figure 5a). For stasis conditions, the flow bypassed while hHSCs were infused for 1 to $3 \mathrm{~min}$ before resuming flow $(25 \mathrm{~mL} / \mathrm{min}$ ), and the cells were not recirculated (only first pass). This procedure was repeated three times and compared to a normal continuous flow set up as has been done previously. We note that the stasis method is of practical significance as it a clinical procedure in current practice and was used to demonstrate the promise of cell therapy in previous clinical trial work utilizing autologous stem cell therapy in acute myocardial infraction. The procedure involved injections of cells through a balloon catheter (normal 'over the wire' PTCA catheter) while the balloon was inflated (occluding flow) for a period of $3 \mathrm{~min}$, delivering a total of 3.3-5 mL of cell suspension. Next the myocardium was perfused by deflating the balloon for 3 min to restore flow in the coronary artery. The process was repeated until the total amount of cells had been delivered. As opposed to the Stasis method, there has been little to no clinical implementation of the continuous flow method in clinical settings since technology was not available to capture cells under continuous shear flow in contrast to the stop and start, stasis method. Given the challenge with implementing the continuous flow method, we compared both using the magnetic stent technology in cell capture.

The number of captured hHSCs and capture efficiency was significantly increased (at least two-fold) using the stasis procedure (Figures $5 \mathrm{~b}$ and $5 \mathrm{c}$ ). This is likely due to the longer time cells spend in proximity to the magnetic stent which facilitate higher efficiency capture. The maximum number of cells retained on ${ }^{n}$ Mag stent (30\% of FePt NPs) was more than 2000 cells $/ \mathrm{mm}^{2}$ (700,000 cells per stent) considering that, like other cells, endothelial progenitor cells can migrate from the outer basolateral surface through the stent struts to proliferate and thus endothelialize the luminal surface as well as the basolateral region. Here, it is observed that cells 
occupy both the luminal and basolateral surface of the stent throughout the entire surface (Figure 5d). Previous work has reported that local delivery (without magnetic localization) of up to $10^{6}$ autologous endothelial progenitor cells in balloon-injured rabbit carotid arteries can result in inhibition of neointimal hyperplasia.

a

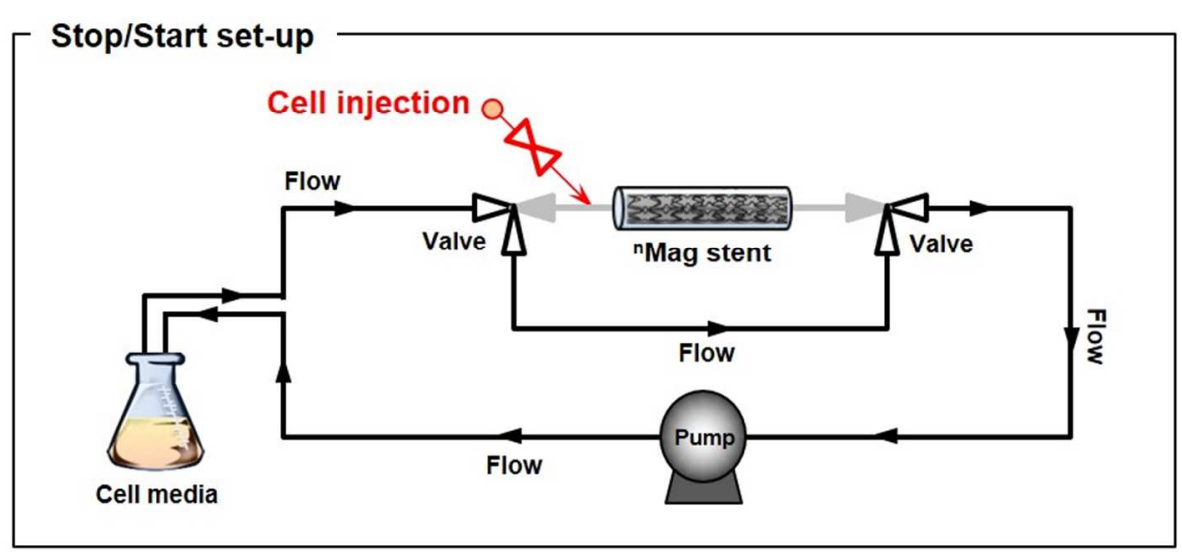

b

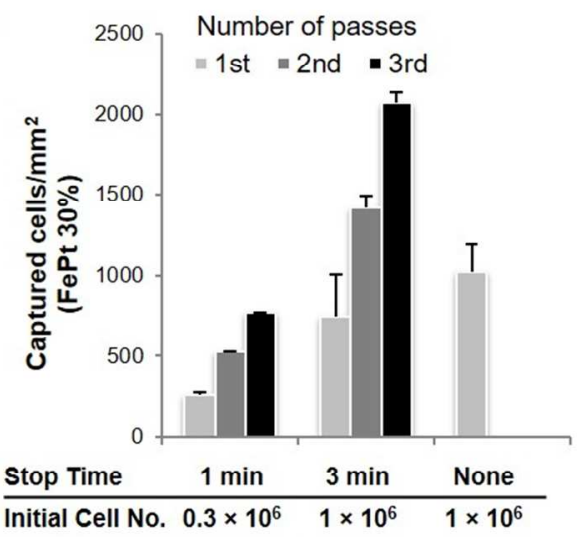

d

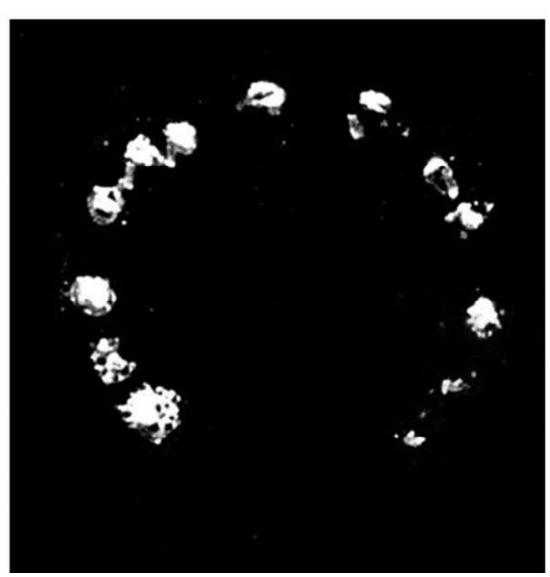

C

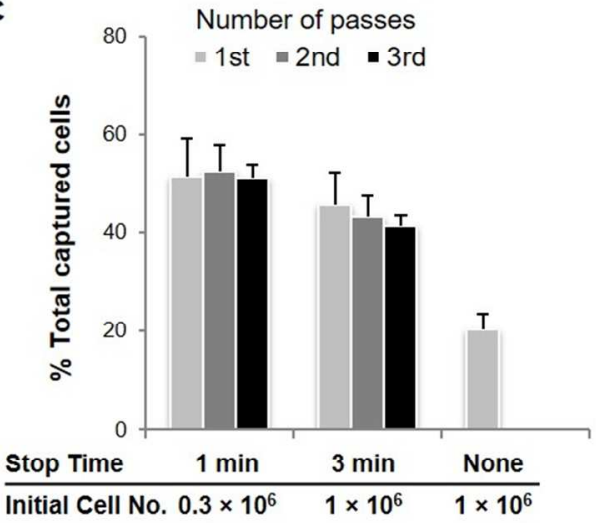

e

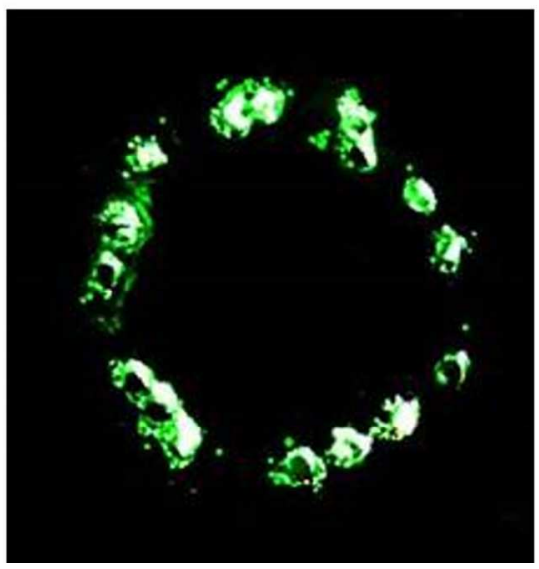


Figure 5. Capture of Human Hematopoetic Stem Cells (hHSCs) (a) Schematic of the "Stop-start stasis/ flow" set-up, a clinically relevant cell administration procedure. Here the flow was stopped and hHSCs were infused for 1 to $3 \mathrm{~min}$ before restarting flow ( $25 \mathrm{~mL} / \mathrm{min})$. The number of hHSCs used was $0.3 \times 10^{6}$ for 1 min stasis and $1.0 \times 10^{6}$ for 3 min stasis set up, respectively. Number of hHSCs captured on ${ }^{\mathrm{n}} \mathrm{Mag}$ stent $\left(\mathrm{FePt} 30 \%\right.$ ) after the $1^{\text {st }}, 2^{\text {nd }}$, and $3^{\text {rd }}$ cell passes depended on the protocol conditions as described. For comparison, $1.0 \times 10^{6}$ cells were continuously infused for $10 \mathrm{~min}$ without holding flow (25 mL/min). (b) Number of cells and (c) capture efficiency (cumulative) are presented. Cross-sections of the ${ }^{n}$ Mag stent (d) before and (e) after cell capture. The coverage of target stem cells along the length of the magnetic stent is represented by fluorescence images (bright field: ${ }^{\mathrm{n}}$ Mag stent struts, green: progenitor stem cells).

Evaluation of ${ }^{\mathrm{n}}$ Mags biodistribution and nanotoxicology. Ultimately, the use of this novel device in vivo, necessitates a rigorous understanding of not only the safety and fate of the device in vivo, but also of that of the individual components. Those components are primarily the ${ }^{n}$ Mags and polymeric coating. For this, we evaluated the biodistribution, clearance, and the acute hematological and organ toxicity of the ${ }^{\mathrm{n}} \mathrm{Mag}$ and PLA composite material in a healthy mouse model. We note that the focus here was on the individual elements and not the entire assembly, since any toxic side-effects or unsafe biodistribution profile would be a direct result of these elements either having an adverse toxic side effect or abnormal biodistribution throughout the body. These studies represent the worst possible scenario in that it assumes the entire device disintegrates into its individual constituents and are circulating in the body. To simulate this condition nanoparticles made from PLA and encapsulating ${ }^{\mathrm{n}}$ Mags were used

First, the ${ }^{n}$ Mag-PLA combination, like other particulate matter, was observed to accumulate in the liver, pancreas, spleen, stomach, and intestines after polymer degradation (Figure S3). The coating material showed peak accumulation in organs at day 2 and was mostly cleared from animals 7 days post systemic administration. A complete blood count panel (CBCs) and clinical chemistries for liver and kidney function were measured at day 1, 7, and 14 days (Figure S4). No significant toxicities were observed in serum measurements of alkaline phosphatase or serum alanine aminotransferase. Normal physiological ranges for mouse alkaline phosphatase are approximately 62-209 IU/L and for alanine aminotransferase approximately 28- 
132 IU/L. No renal toxicity was observed, as blood urea nitrogen levels were within the normal mouse reference range of $18-29 \mathrm{mg} / \mathrm{dL}$. Complete blood counts demonstrated that normal physiological ranges leukocyte counts, platelet counts, and hemoglobin content.

To ascertain levels of acute cytokines that may be induced as a result of the material, bone marrow-derived macrophages (BMMs) harvested from C57BL/6 mice were assessed for secretion of TNF- $\alpha$, IFN $\gamma$, and IL-4. IL-4 was measured as a proxy for potential allergic responses, IFN $\gamma$ and TNF- $\alpha$ for inflammatory responses (Figure S5). Generally, the coating material was non-inflammatory and neither the particles nor polymer showed signs or instigated an allergic response. An elevated secretion of IFN $\gamma$ was noted only at a high ${ }^{\mathrm{n}}$ Mag-PLA dose of $1 \mathrm{mg} / \mathrm{mL}$.

In this work we demonstrated a novel, non-incremental and significant advance in technology that may solve issues related to the long-term complications with stent implantation. The key new feature is a strong intrinsic magnetic capability engineered in a metallic nonferromagnetic stent made from Magnesium, a bioabsorbable metal with well-known and demonstrated benefits in stent manufacture, and applications in tissue engineering and regenerative medicine. Yet, despite its promise as a bioabsorbable metal with attractive mechanical properties for stent fabrication, early and long-term intravascular and angiographic studies in human trials with $\mathrm{Mg}$ stents showed that the bare metal stents alone do not inhibit development of restenosis in the first few months after implantation. ${ }^{38}$ In human clinical trials, it was found that the rate of stent degradation, which proved to be too fast, compromise the stent radial strength leading to recoiling early after arterial deployment and ultimately playing a significant role in the development restenosis and late term thrombosis. Such complications are alleviated if endothelialization was made to happen early after implantation. Just using the bare metal stent, endothelialization was observed as early as 1 month after implantation. ${ }^{38}$ Given that this work focuses on inducing rapid capture of injected cells then one would expect that the degradation rate of the bare metal stent will play a smaller role since the tissue regenerative process is expedited, thereby abrogating any advanced side-effects due to implant presence. As such, our technology obviates new structural designs or the search for Mg alloys that may slow down the degradation process. It may also obviate the need for drug regimens either administered systemically or locally through drug eluting stents. With this technology, tissue 
regeneration would occur faster relative to any potential mechanical failure and while, we did not encapsulate a drug in the polymer coating, the current design does accommodate for such an addition, if deemed attractive for a particular stent application. In summary, this newer design offers several critical features that make it an alternative to current gold standards: 1) A safe toxicological profile of the magnetic nanoparticle and polymer components together with rapid clearance of the $\mathrm{Np}$ from the body. 2) the elimination of the requirement for strong external magnets, such as MRI solenoids in the operating room, as would be needed for a paramagnetic stent. 3) The properties of the Mg metal itself; showing excellent mechanical and biological properties that minimize inflammation, neutralize the acidic polymer products, and degradative properties that were no doubt key in why this material was chosen for testing in humans. Therefore, this study adds additional attractive features to the bare metal stent used in man and these features promise to solve the issues that have limited durable and long-term stent therapeutic outcomes.

\section{Methods.}

Fabrication of the Magnetic nanoparticles (" $\left.{ }^{\mathbf{n}} \mathrm{Mag}\right)$. The bare metal stent was coated with magnetic nanoparticles (FePt NPs) embedded in a biodegradable polymer (PLA). Synthesis of FePt np involved simultaneous chemical reduction of $\mathrm{Pt}(\mathrm{acac})_{2}$ and $\mathrm{Fe}(\mathrm{acac})_{3}$ (See supplementary information) and a previous report that was the basis of the current fabrication process. ${ }^{33}$ FePt NPs exhibited maximal coercivity when the composition was a molar ratio of 1:1 (Fe:Pt). The synthesized FePt NPs were coated with $\mathrm{SiO}_{2}$ by base-catalyzed silica formation using tetraethylorthosilicate (TEOS) to minimize thermal sintering of FePt particles. ${ }^{34}$ Detailed protocols are in the supplementary information. The $\mathrm{SiO}_{2}$ coated FePt particles were annealed in a tube furnace (three Zones Split Quartz Tube Furnace, MTI Co.) for $10 \mathrm{~min}$ at $650^{\circ} \mathrm{C}$ under a forming gas flow with the following composition $\left(7 \% \mathrm{H}_{2} / 93 \% \mathrm{~N}_{2}\right)$. Next, the silica coating was completely removed by washing the particles with HF (1\%) in deionized (DI) water three times followed by a DI water wash twice.

Coating of the Magnesium stent with Magnetic nanoparticles ( ${ }^{\mathrm{n}}$ Mag-Mg Stent). Bare metal Mg stents with a strut thickness of $100 \mu \mathrm{m}$ were coated with PLA- ${ }^{\mathrm{n}}$ Mag mixture (PLA: 
LACTEL $^{\circledR}$, inherent viscosity $0.15-0.35 \mathrm{dL} / \mathrm{g}$, Durect co.) $1 \mathrm{mg} / \mathrm{mL}$ in $\mathrm{CHCl}_{3}$. The PLA to nMag ratio by wt was $30 \%$. The mixture was electrosprayed ${ }^{39}$ over $\mathrm{Mg}$ stents in the 'cone jet' mode using ElectroNanospray (Nanocopoeia Inc. ${ }^{\mathrm{TM}}$, rotation speed: $30 \mathrm{rpm}$, flowrate: 0.03 $\mathrm{mL} / \mathrm{min}$, injection volume: $250 \mu \mathrm{L}$ ) to create a $30 \mu \mathrm{m}$ PLA coating. Magnetization was induced in the dried coated stents by inserting the coated stents in the bore of a Bruker MRI solenoid (1.5T) overnight.

A rough approximation of the magnetic field in the stent. Magnetic field $\left(\mathrm{B}^{*}\right)$ was generated by superimposing the individual fields of axially-aligned dipoles of ${ }^{\mathrm{n}}$ Mags that were symmetrically located inside a cylindrical tube (stent) with a length (L) to radius (R) ratio of 9 (18mm long $\mathrm{x} 4 \mathrm{~mm}$ diameter):

$$
B^{*}(r)=\sum_{j=1}^{N}\left[\frac{3 r_{j}\left(m \cdot r_{j}\right)}{\left|r_{j}\right|}-\frac{m}{\left|r_{j}\right|^{3}}\right]
$$

Where $r_{j}$ is distance vector from the jth dipole to the point of interest (r) and the unit magnetic moments $(\mathrm{m})$ of all the dipoles point in the axial direction.

Cell labeling for capture efficiency experiments. State all cells used here. were stained with carboxyfluorscein succinmide ester (CFSE) for tracking (supplementary information). Stained cells were then labeled with iron oxide) by one of two methods. Method 1: Surface labeling of hHSCs using CliniMACS (a dextran-coated IO agent) immobilized with anti-CD34 antibodies followed by the protocol from manufacturer (supplementary information). Method 2: Intracellular labeling cells with IO. IO NPs $(100 \mu \mathrm{g} / \mathrm{mL})$ were incubated with macrophages and HUVECs in culture at $37^{\circ} \mathrm{C}$ for $2 \mathrm{~h}$.

Two flow protocols were used. Impact of flow conditions, ${ }^{n}$ Mag seeding density, and number of infused cells on the efficiency of magnetic cell capture was investigated. To ascertain the efficacy of cell capture by the magnetized stent, we built a flow bioreactor set up that was operated in stasis or continuous modes as shown in Figure 5a under standard cell culture 
conditions $\left(37^{\circ} \mathrm{C}\right.$ and $5 \% \mathrm{CO}_{2}$ ). $\mathrm{Mg}$ stents coated with a PLA layer containing $0,5,15$, and $30 \%$ ${ }^{\mathrm{n}} \mathrm{Mag}$ was deployed in transparent silicon tubing (4 $\mathrm{mm}$ inner diameter) connected via flexible tubes to a liquid reservoir and a peristaltic pump (Masterflex ${ }^{\circledR}$, Cole-Parmer Ins.). Cell media was circulated at 25 or $50 \mathrm{~mL} / \mathrm{min}$ and 0.3 or $1 \times 10^{6}$ of labeled cells were injected $3 \mathrm{~cm}$ away from the stent, while media continuously flew. For stasis flow control, flow was paused while cells were injected and resumed the flow. The cells were injected three times by dividing them into three parts (each injection: 0.1 and $0.33 \times 10^{6}$ cells were injected for 1 and $3 \mathrm{~min}$, respectively). To quantify number of captured cells, stents were imaged by a Bruker molecular imaging instrument (Carestream Health, Inc.) at the desired time points after washing them with a cell-free media. A calibration standard of fluorescently labeled cells was used to calculate actual number of cells captured. Degradation study of ${ }^{\mathrm{n}} \mathrm{Mag}$ stent was performed in Dulbecco's Modified Eagle Medium (DMEM) in a flow chamber at the flow rate of $25 \mathrm{~mL} / \mathrm{min}$. 


\section{References}

1. Analysis, M. T. M., Cardiovascular Surgical and Interventional Procedures to 2022. http://mediligence.com/c500/, 2016.

2. $\quad$ Flight, M. H. Nature reviews. Drug discovery 2011, 10, (12), 902.

3. Merkely, B.; Toth-Zsamboki, E.; Becker, D.; Beres, B. J.; Szabo, G.; Vargova, K.; Fulop, G.; Kerecsen, G.; Preda, I.; Spaulding, C.; Kiss, R. G. Can J Cardiol 2009, 25, (4), 229-232.

4. Schmidt, M.; Pedersen, L.; Maeng, M.; Lassen, J. F.; Lash, T. L.; Nielsen, T. T.; Sorensen, H. T. Pharmacotherapy 2011, 31, (5), 458-468.

5. Lim, G. B. Nature reviews. Cardiology 2015, 12, (10), 559.

6. McFadden, E. P.; Stabile, E.; Regar, E.; Cheneau, E.; Ong, A. T. L.; Kinnaird, T.; Suddath, W. O.; Weissman, N. J.; Torguson, R.; Kent, K. M.; Pichard, A. D.; Satler, L. F.; Waksman, R.; Serruys, P. W. Lancet 2004, 364, (9444), 1519-1521.

7. $\quad$ Park, D. W.; Park, S. W.; Park, K. H.; Lee, B. K.; Kim, Y. H.; Lee, C. W.; Hong, M. K.; Kim, J. J.; Park, S. J. Am J Cardiol 2006, 98, (3), 352-356.

8. $\quad$ Ladich, E.; Nakazawa, G.; Cook, S.; Windecker, S.; Burke, A.; Kolodgie, F.; Virmani, R. Circulation 2008, 118, (18), S1047-S1047.

9. Mehran, R.; Dangas, G.; Abizaid, A. S.; Mintz, G. S.; Lansky, A. J.; Satler, L. F.; Pichard, A. D.; Kent, K. M.; Stone, G. W.; Leon, M. B. Circulation 1999, 100, (18), 1872-8.

10. VanBelle, E.; Tio, F. O.; Couffinhal, T.; Maillard, L.; Passeri, J.; Isner, J. M. Circulation 1996, 94, (8), 4073-4073.

11. Sprague, E. A.; Luo, J.; Palmaz, J. C. J Vasc Interv Radiol 1997, 8, (1), 83-92.

12. Motwani, M. S.; Rafiei, Y.; Tzifa, A.; Seifalian, A. M. Biotechnol Appl Bioc 2011, 58, (1), 2-13.

13. Dichek, D. A.; Neville, R. F.; Zwiebel, J. A.; Freeman, S. M.; Leon, M. B.; Anderson, W. F. Circulation 1989, 80, (5), 1347-53.

14. Scott, N. A.; Candal, F. J.; Robinson, K. A.; Ades, E. W. American heart journal 1995, $129,(5), 860-6$.

15. Herring, M.; Gardner, A.; Glover, J. Surgery 1978, 84, (4), 498-504.

16. Belden, T. A.; Schmidt, S. P.; Falkow, L. J.; Sharp, W. V. Transactions - American Society for Artificial Internal Organs 1982, 28, 173-7.

17. Ortenwall, P.; Wadenvik, H.; Kutti, J.; Risberg, B. Journal of vascular surgery 1990, 11, (3), 403-10.

18. Pislaru, S. V.; Harbuzariu, A.; Gulati, R.; Witt, T.; Sandhu, N. P.; Simari, R. D.; Sandhu, G. S. Journal of the American College of Cardiology 2006, 48, (9), 1839-45.

19. Uthamaraj, S.; Tefft, B. J.; Hlinomaz, O.; Sandhu, G. S.; Dragomir-Daescu, D. Jove-J Vis $\operatorname{Exp} 2015,(103)$.

20. Konig, A.; Schiele, T. M.; Rieber, J.; Theisen, K.; Mudra, H.; Klauss, V. Z Kardiol 2002, 91 Suppl 3, 98-102.

21. Mao, L.; Shen, L.; Chen, J. H.; Zhang, X. B.; Kwak, M.; Wu, Y.; Fan, R.; Zhang, L.; Pei, J.; Yuan, G. Y.; Song, C. L.; Ge, J. B.; Ding, W. J. Sci Rep-Uk 2017, 7.

22. Tarnawski, A.; Erickson, R. A.; Durbin, T.; Chang, K. Gastroenterology 1994, 106, (4), A194-A194.

23. Wheatley, R. G.; Kallus, F. T.; Reynolds, R. C.; Giesecke, A. H. Anesthesiology 1979, 50, (6), 514-519. 
24. Brown, A.; Zaky, S.; Ray, H.; Sfeir, C. Acta Biomater 2015, 11, 543-553.

25. Chen, Z. T.; Mao, X. L.; Tan, L. L.; Friis, T.; Wu, C. T.; Crawford, R.; Xiao, Y. Biomaterials 2014, 35, (30), 8553-8565.

26. Lee, H. W.; Seo, S. H.; Kum, C. H.; Park, B. J.; Joung, Y. K.; Son, T. I.; Han, D. K. Macromol Res 2014, 22, (2), 210-218.

27. Gu, X. N.; Zheng, Y. F.; Cheng, Y.; Zhong, S. P.; Xi, T. F. Biomaterials 2009, 30, (4), 484-498.

28. Heublein, B.; Rohde, R.; Kaese, V.; Niemeyer, M.; Hartung, W.; Haverich, A. Heart 2003, 89, (6), 651-656.

29. Chaya, A.; Yoshizawa, S.; Verdelis, K.; Myers, N.; Costello, B. J.; Chou, D. T.; Pal, S.; Maiti, S.; Kumta, P. N.; Sfeir, C. Acta Biomater 2015, 18, 262-269.

30. Hans, C. P.; Chaudhary, D. P.; Bansal, D. D. Magnesium Res 2003, 16, (1), 13-19.

31. Cobbold, M.; Keenan, R.; Khan, N.; McDonald, D.; Mahendra, P.; Craddock, C.; Moss, P. Blood 2001, 98, (11), 852a-852a.

32. Baglin, J. E. E.; Sun, S. H.; Kellock, A. J.; Thomson, T.; Toney, M. F.; Terris, B. D.; Murray, C. B. Mater Res Soc Symp P 2003, 777, 53-58.

33. Usov, N. A.; Barandiaran, J. M. Appl Phys Lett 2012, 101, (17).

34. Wu, X. W.; Guslienko, K. Y.; Chantrell, R. W.; Weller, D. Appl Phys Lett 2003, 82, (20), 3475-3477.

35. Arai, M.; Miyake, M.; Yamada, M. J Phys Chem C 2008, 112, (6), 1953-1962.

36. Elkins, K. E.; Vedantam, T. S.; Liu, J. P.; Zeng, H.; Sun, S. H.; Ding, Y.; Wang, Z. L. Nano Lett 2003, 3, (12), 1647-1649.

37. Lee, D. C.; Mikulec, F. V.; Pelaez, J. M.; Koo, B.; Korgel, B. A. The journal of physical chemistry. B 2006, 110, (23), 11160-6.

38. Waksman, R.; Erbel, R.; Di Mario, C.; Bartunek, J.; de Bruyne, B.; Eberli, F. R.; Erne, P.; Haude, M.; Horrigan, M.; Ilsley, C.; Bose, D.; Bonnier, H.; Koolen, J.; Luscher, T. F.; Weissman, N. J.; Investigators, P.-A. JACC Cardiovasc Interv 2009, 2, (4), 312-20.

39. Puskas, J. E.; Munoz-Robledo, L. G.; Hoerr, R. A.; Foley, J.; Schmidt, S. P.; EvanchoChapman, M.; Dong, J. P.; Frethem, C.; Haugstad, G. Wires Nanomed Nanobi 2009, 1, (4), 451462. 